\begin{tabular}{c|c|c|} 
SELECCIONES MATEMÁTICAS \\
Universidad Nacional de Trujillo \\
ISSN: $2411-1783$ (Online) \\
2021; Vol. 8(1): 131-146.
\end{tabular}

\title{
Stability in Kolmogorov-type quadratic systems describing interactions among two species. A brief revision
}

\section{Estabilidad en sistemas cuadráticos del tipo Kolmogorov describiendo interacciones entre dos especies. Una breve revisión}

\author{
Eduardo González-Olivares (i) and Alejandro Rojas-Palma (i)
}

Received, Mar. 27, 2021

Accepted, Jun. 29, 2021

How to cite this article:

González-Olivares E, Rojas-Palma A. Stability in Kolmogorov-type quadratic systems describing interactions among two species. A brief revision. Selecciones Matemáticas. 2021;8(1):131-146. http://dx.doi .org/10.17268/ sel.mat.2021.01.13

\begin{abstract}
Population dynamics is a relevant topic in Biomathematics, being the study of the long-term behavior of interaction models between species, one of its central problems. A large part of these relationships are described by ordinary differential equations (ODE), having as main objectives the study of the stability of their solutions.

In this document we mainly describe the dynamic behavior of the Volterra predation model. In addition, we make a review of some derived predation models and a brief review of the dynamical properties of models describing other interactions between species such as: competition, mutualism, amensalism, and commensalism; also described by nonlinear ODE systems of the second order of Kolmogorov-type.

For each of these models, the non-existence of limit cycles can be demonstrated and in most of them, there is a globally stable equilibrium point. In one of them, there are conditions in the parameters for which the only positive equilibrium point is a center, as in the original Lotka-Volterra model.

The methodology used is the usual one for the analysis of models with hyperbolic equilibrium points, but it can guide the analysis of other more complicated models.
\end{abstract}

Keywords . Predator-prey model, functional response, cycles, separatrix curve, stability, Lyapunov function

\section{Resumen}

La dinámica de poblaciones es un tema relevante en Biomatemática, siendo el estudio del comportamiento a largo plazo de los modelos de interacción entre especies, uno de sus problemas centrales. Gran parte de estas relaciones son descritas por sistemas de ecuaciones diferenciales ordinarias (EDO), teniendo como objetivos principales el estudio de la estabilidad de las soluciones.

En este documento describimos principalmente el comportamiento dinámico del modelo de depredación de Volterra. Además, hacemos una revisión de algunos modelos de depredación derivados y una breve reseña de las propiedades dinámicas de modelos describiendo otras interacciones entre especies tales como: competencia, mutualismo, amensalismo y comensalismo, también descritos por sistemas EDO no lineales del tipo Kolmogorov de segundo orden.

El principal resultado obtenido para cada uno de estos modelos es la no existencia de ciclos límites; sin embargo, en uno de ellos existen condiciones en los parámetros para los cuales el único punto de equilibrio positivo es un centro, como sucede en el modelo original de Lotka-Volterra.

La metodología empleada en este trabajo es la usual para el análisis de modelos con puntos de equilibrio hiperbólicos, pero puede orientar el análisis de otros modelos más complicados.

\footnotetext{
*Pontificia Universidad Católica de Valparaíso, Chile. (e jgonzal@ucv . cl).

†Departamento de Matemática, Física y Estadística, Facultad de Ciencias Básicas, Universidad Católica del Maule, Talca, Chile. (amrojas@ucm.cl).
} 
Palabras clave. Modelo depredador-presa, respuesta funcional, ciclos, curva separatriz, estabilidad, función de Lyapunov

1. Introducción. El modelo de Volterra es descrito por el sistema autónomo bidimensional de ecuaciones diferenciales del tipo Kolmogorov [12, 14]:

$$
X_{\eta}(x, y):\left\{\begin{aligned}
\frac{d x}{d t} & =\left(r\left(1-\frac{x}{K}\right)-q y\right) x \\
\frac{d y}{d t} & =(p x-c) y
\end{aligned}\right.
$$

donde $x=x(t)$ e $y=y(t)$, representan los tamaños poblacionales de presas y depredadores, respectivamente, para $t \geq 0$ (medido en biomasa, número de individuos o densidad); los parámetros son todos positivos, esto es, $\eta=(r, K, q, p, c) \in \mathbb{R}_{+}^{5}$, teniendo los siguientes significados ecológicos:

$r$ es la tasa de crecimiento intrínseco o potencial biótico de las presas;

$K$ es la capacidad de soporte del medio ambiente para las presas;

$q$ es la tasa de consumo de los depredadores;

$p$ es el coeficiente de conversión de biomasa de presas en el nacimiento de nuevos depredadores, $\mathrm{y}$

$c$ es la tasa natural de muerte de los depredadores en ausencia de la presas.

El sistema (1.1) está definido en el primer cuadrante

$$
\Omega=\left\{(x, y) \in \mathbb{R}^{2} / x \geq 0, y \geq 0\right\}=\left(\mathbb{R}_{0}^{+}\right)^{2} .
$$

En el ámbito de la Bioeconomía Matemática el sistema (1.1) se denomina modelo de Smith [7, 15].

\section{Puntos de equilibrio}

Los puntos críticos o estacionarios del sistema se obtienen considerando que $\frac{d x}{d t}=0=\frac{d x}{d t}$.

Los sistemas algebraicos son

$$
\left\{\begin{array}{l}
x=0 \\
y=0
\end{array},\left\{\begin{array}{r}
r\left(1-\frac{x}{K}\right)-q y=0 \\
y=0
\end{array} \text { y } \begin{array}{rl}
r\left(1-\frac{x}{K}\right)-q y & =0 \\
p x-c & =0
\end{array} .\right.\right.
$$

Los puntos de equilibrio son: el estado de extinción $(0,0)$, el estado de exclusión de los depredadores $(K, 0)$ y un equilibrio de coexistencia $\left(x_{e}, y_{e}\right)$ el cual corresponde a la intersección de las isoclinas (curvas de igual inclinación):

$$
y=\frac{r}{q}\left(1-\frac{x}{K}\right) \text { y } p x-c=0 .
$$

Entonces, $x_{e}=\frac{c}{p}$ e $y_{e}=\frac{r}{q}\left(1-\frac{c}{p K}\right)$.

Claramente, $\left(x_{e}, y_{e}\right)$ está en el interior del primer cuadrante o es un equilibrio positivo, si sólo si, $1-\frac{c}{p K}>0$, esto es, si sólo si, $p K-c>0$.

Observamos que los puntos $\left(x_{e}, y_{e}\right)$ y $(K, 0)$ coinciden, si y sólo si, $p K-c=0$.

\section{Matriz Jacobiana}

Para analizar la naturaleza local de los puntos de equilibrio hiperbólicos o genéricos, de acuerdo al Teorema de Hartman y Grossman (o Teorema de Linealización) [6, 11, 26], se debe analizar la matriz Jacobiana [6] (o matriz de la comunidad [30]) dada por

$$
D X_{\eta}(x, y)=\left(\begin{array}{cc}
-\frac{1}{K}(2 r x-K r+K q y) & -q x \\
p y & p x-c
\end{array}\right),
$$

donde,

$$
\begin{aligned}
& \operatorname{det} D X_{\eta}(x, y)=\frac{-2 p r x^{2}+(2 c r+K p r) x+(K c q y-K c r)}{K}, \mathrm{y} \\
& \operatorname{tr} D X_{\eta}(x, y)=-\frac{(2 r-K p) x+(K c-K r+K q y)}{K} .
\end{aligned}
$$

1.1. Resultados principales. A continuación describimos las principales propiedades del sistema (1.1)

te

Lema 1.1. El conjunto $\Gamma=\{(x, y) \in \Omega / 0 \leq x \leq K, y \geq 0\}$ es una región positivamente invarian-

Demostración: Claramente los ejes coordenados son conjuntos invariantes [6], pues el sistema es del tipo Kolmogorov [12].

Considerando $x=K$, se tiene que $\frac{d x}{d t}=-q K y<0$ y cualquiera sea el signo de $\frac{d y}{d t}$, las trayectorias entran a la región $\Gamma$.

Lema 1.2. Las soluciones son uniformemente acotadas 
Demostración: Usando Teorema de comparación para desigualdades diferenciales, (ver página 29 [3]). De la primera ecuación del sistema (1.1) tiene

teniendo que

$$
\frac{d x}{d t} \leq r\left(1-\frac{x}{K}\right) x, \forall x \in \mathbb{R}_{+}^{2},
$$

$$
\begin{aligned}
& x(t) \rightarrow K, \text { when } t \rightarrow \infty \text { and } x>0 . \\
& x(t) \rightarrow K, \text { when } t \rightarrow \infty \text { and } x>K .
\end{aligned}
$$

Considerando $P=$ máx $\{x(0), K\}$, de la desigualdad anterior tenemos

$$
x(t) \leq P, \forall t \geq 0 .
$$

Sea $W=a x+b y$ con $a$ y $b$ parámetros por determinar.

Claramente $0<W(t), \forall t \geq 0$.

Derivando $W(t)$ respecto a $t$, obtenemos

$$
\begin{aligned}
\frac{d W}{d t} & =a \frac{d x}{d t}+b \frac{d y}{d t}, \\
& =a\left(r\left(1-\frac{x}{K}\right)-q y\right) x+b(p x-c) y,
\end{aligned}
$$

Escogiendo $b=\frac{a q}{p}$, se llega a

$$
\frac{d W}{d t}=\operatorname{ar}\left(1-\frac{x}{K}\right) x-\frac{a q}{p} c y
$$

Considering $\left(A+u^{n}\right) \leq(A+P)$, let us $L=B(A+P)$.

Por lo tanto,

$$
\begin{aligned}
\frac{d W}{d t}+c W & =a r\left(1-\frac{x}{K}\right) x-\frac{a q}{p} c y+a c x+\frac{a q}{p} c y, \\
\frac{d W}{d t}+c W & =\left(a r+a c-\frac{a r x}{K}\right) x, \\
\frac{d W}{d t}+c W & \leq(a r+a c) x \\
\frac{d W}{d t}+c W & \leq a(r+c) P .
\end{aligned}
$$

Aplicando el teorema de comparación para desigualdades diferenciales (página 30 en [3]), obtenemos

$$
W \leq N e^{-c t}+\frac{a}{c}(r+c) P, \text { siendo } N=e^{M c} .
$$

Cuándo, $t \rightarrow \infty$, entonces, $W \leq \frac{a}{c}(r+c) P$.

Por tanto, las soluciones del sistema (1.1) están acotadas.

Esta propiedad implica que el modelo está bien propuesto (well-posed) [2], pues carece de sentido ecológico que existan muy pocas presas y una cantidad muy grande de depredadores especialistas, sin que se extinga esta segunda población.

1.1.1. Naturaleza de los puntos sobre los ejes. Para los puntos de equilibrio $(0,0)$ y $(K, 0)$ se tiene: Lema 1.3. a) El punto $(0,0)$ es punto silla para todo valor de parámetro.

b) El punto $(K, 0)$ es:

i) una silla hiperbólica, si y sólo si, $p K-c>0$.

ii) un atractor hiperbólico, si y sólo si, $p K-c<0$.

iii) un silla-nodo atractor [6, 11], si y sólo si, $p K-c=0$.

Demostración: a) La evaluación de la matriz Jacobiana en $(0,0)$ es

$$
D X_{\eta}(0,0)=\left(\begin{array}{cc}
r & 0 \\
0 & -c
\end{array}\right),
$$

y claramente, $\operatorname{det} D X_{\eta}(0,0)<0$. Por lo tanto, usando el Teorema de la traza y el determinante [24], el punto $(0,0)$ es punto silla.

b) La evaluación de la matriz Jacobiana en $(K, 0)$ es

$$
D X_{\eta}(K, 0)=\left(\begin{array}{cc}
-r & -q K \\
0 & p K-c
\end{array}\right) ;
$$

entonces, $(K, 0)$ es:

i) una silla hiperbólica, si y sólo si, $p K-c>0$, pues $\operatorname{det} D X_{\eta}(K, 0)=-r(p K-c)<0$,

ii) un atractor, si y sólo si, $p K-c<0$, pues $\operatorname{det} D X_{\eta}(K, 0)=-r(p K-c)>0$ y $\operatorname{tr} D X_{\eta}(K, 0)=$ $-r+(p K-c)<0$.

iii) un silla-nodo atractor, si y sólo si, $p K-c=0$, pues $\operatorname{det} D X_{\eta}(K, 0)=-r(p K-c)=0$. En este caso el punto $\left(x_{e}, y_{e}\right)$ coincide con $(K, 0)$. 
1.1.2. Naturaleza del punto de equilibrio positivo. La estabilidad del punto de equilibrio al interior del primer cuadrante es dada a continuación

Teorema 1.1. El punto de equilibrio $\left(x_{e}, y_{e}\right)$ es:

a) un atractor local, si y sólo si, $p K-c>0$.

b) un silla-nodo atractor, si y sólo si, $p K-c=0$.

Demostración: La evaluación de la matriz Jacobiana el punto $\left(x_{e}, y_{e}\right)$ es

$$
D X_{\eta}\left(x_{e}, y_{e}\right)=\left(\begin{array}{cc}
-\frac{1}{K} r x_{e} & -q x_{e} \\
p y_{e} & 0
\end{array}\right) \text {. }
$$

Entonces, $\left(x_{e}, y_{e}\right)$ es

a) un punto de equilibrio atractor, , si y sólo si, $p K-c>0$, pues

$$
\operatorname{det} D X_{\eta}\left(x_{e}, y_{e}\right)=p q x_{e} y_{e}>0 \mathrm{y} \operatorname{tr} D X_{\eta}\left(x_{e}, y_{e}\right)=-\frac{1}{K} r x_{e}<0 .
$$

b) Es un silla-nodo atractor si y sólo si, $p K-c=0$.

Observación 1.1. El punto $\left(x_{e}, y_{e}\right)$ está en fuera del primer cuadrante, si y sólo si, $p K-c<0$. En tal caso, aplicando el Teorema de Poincaré-Bendixson [13] y sabiendo que las soluciones son acotadas en $\Gamma$, el punto $(K, 0)$ es atractor global asintóticamente estable, en el sentido que

$$
\lim _{t \rightarrow \infty} x(t)=K \text { y } \lim _{t \rightarrow \infty} y(t)=0 .
$$

En forma análoga, cuando el punto $\left(x_{e}, y_{e}\right)$ está en el interior del primer cuadrante, es un atractor global asintóticamente estable aplicando el teorema de Poincaré-Bendixson [13].

Para demostrar la estabilidad global del punto de equilibrio puede construirse una función de Lyapunov [14]. Además, podemos excluir la existencia de órbitas periódicas usando el criterio de BendixsonDulac [8].

Teorema 1.2. El punto de equilibrio $\left(\frac{c}{p}, y_{e}\right)$ es globalmente asintóticamente estable, si y sólo si, $p K-c>0$.

Demostración: Usando la función de Lyapunov propuesta por Goh [14]

$$
V(x, y)=c_{1}\left(x-x_{e}-x_{e} \ln \frac{x}{x_{e}}\right)+c_{2}\left(y-y_{e}-y_{e} \ln \frac{y}{y_{e}}\right)
$$

se tiene que al derivada de $V$ respecto a $t$ es:

$$
\frac{d V}{d t}(x, y)=c_{1}\left(\frac{d x}{d t}-x_{e} \frac{1}{x} \frac{d x}{d t}\right)+c_{2}\left(\frac{d y}{d t}-y_{e} \frac{1}{y} \frac{d y}{d t}\right)
$$

Factorizando se tiene:

$$
\frac{d V}{d t}(x, y)=c_{1}\left(x-x_{e}\right) \frac{1}{x} \frac{d x}{d t}+c_{2}\left(y-y_{e}\right) \frac{1}{y} \frac{d y}{d t}
$$

Reemplazando cada factor se obtiene

$$
\frac{d V}{d t}(x, y)=c_{1}\left(x-x_{e}\right)\left(r\left(1-\frac{x}{K}\right)-q y\right)+c_{2}\left(y-y_{e}\right)(p x-c) .
$$

Como el punto de equilibrio satisface la ecuación de las isoclinas se tiene que:

$$
\begin{aligned}
\frac{d V}{d t}(x, y) & =c_{1}\left(x-x_{e}\right)\left(\left(r\left(1-\frac{x}{K}\right)-q y\right)-\left(r\left(1-\frac{x_{e}}{K}\right)-q y_{e}\right)\right) \\
& +c_{2}\left(y-y_{e}\right)\left((p x-c)-\left(p x_{e}-c\right)\right) .
\end{aligned}
$$

Luego, agrupando se obtiene que

$$
\frac{d V}{d t}(x, y)=c_{1}\left(x-x_{e}\right)\left(-r \frac{x}{K}+r \frac{x_{e}}{K}-q y+q y_{e}\right)+c_{2}\left(y-y_{e}\right)\left(p\left(x-x_{e}\right)\right)
$$

o bien,

$$
\begin{gathered}
\frac{d V}{d t}(x, y)=c_{1}\left(x-x_{e}\right)\left(-\frac{r}{K}\left(x-x_{e}\right)-q\left(y-y_{e}\right)\right)+c_{2} p\left(y-y_{e}\right)\left(x-x_{e}\right), \\
\frac{d V}{d t}(x, y)=c_{1}\left(x-x_{e}\right)\left(-\frac{r}{K}\left(x-x_{e}\right)\right)-c_{1}\left(x-x_{e}\right) q\left(y-y_{e}\right)+c_{2} p\left(y-y_{e}\right)\left(x-x_{e}\right) .
\end{gathered}
$$


Eligiendo $c_{2}=-\frac{c_{1} q}{p}$, se obtiene que

$$
\frac{d V}{d t}(x, y)=-\frac{r}{K} c_{1}\left(x-x_{e}\right)^{2}<0
$$

satisfaciendo la definición de una función de Lyapunov [14].

En consecuencia, el punto $\left(\frac{c}{p}, y_{e}\right)$ es global asintóticamente estable, es decir, es el $\omega$-limite de todas las trayectorias del sistema.

Teorema 1.3. En el sistema (1.1) no existen órbitas periódicas o ciclos.

Demostración: Usando Test o criterio de Bendixson Dulac [8, 10].

Consideremos la función $g(x, y)=\frac{1}{x y}$

Debemos determinar el signo de la expresión, siendo

$$
H(x, y)=\frac{\partial}{\partial x}(P(x, y) g(x, y))+\frac{\partial}{\partial y}(Q(x, y) g(x, y)) \text {, }
$$

$$
P(x, y)=\left(r\left(1-\frac{x}{K}\right)-q y\right) x \text { y } Q(x, y)=(p x-c) y .
$$

Luego, tenemos

$$
\begin{aligned}
& H(x, y)=\frac{\partial}{\partial x}\left(\left(r\left(1-\frac{x}{K}\right)-q y\right) \frac{1}{y}\right)+\frac{\partial}{\partial y}\left((p x-c) \frac{1}{x}\right) \\
& =-\frac{1}{K} \frac{r}{y}<0 .
\end{aligned}
$$

Por lo tanto no existen órbitas periódicas o ciclos.

1.1.3. Comportamiento al infinito. Un resultado importante que hemos demostrado es la existencia de una región de invarianza $\Gamma$ (Lema 1.1), mostrando que hay soluciones que ingresan a $\Gamma$. Pero surge la pregunta, ¿Cual es el $\alpha$-límite de esas trayectorias del sistema?

Para responder esa cuestión estudiaremos el comportamiento al infinito, analizando el punto $(\infty, 0)$, en el dominio compacto de $\Omega$.

Teorema 1.4. El punto $(\infty, 0)$ en el compacto de $\Omega$ es un punto silla

Demostración: Consideremos la compactificación de Poincaré [26], dada por la transformación:

$$
x=\frac{1}{X} \text { e } y=\frac{Y}{X} \text {, o bien, } X=\frac{1}{x} \text { e } Y=\frac{y}{x} .
$$

Notamos que si $x \rightarrow \infty$, entonces $X$ e $Y \rightarrow 0$.

Luego, $\frac{d x}{d t}=\frac{d x}{d X} \frac{d X}{d t}=-\frac{1}{X^{2}} \frac{d X}{d t}$ y $\frac{d y}{d t}=-\frac{Y}{X^{2}} \frac{d X}{d t}+\frac{1}{X} \frac{d Y}{d t}$.

Obtenemos el nuevo sistema

es decir,

$$
Z_{\eta}\left((X, Y):\left\{\begin{array}{rl}
-\frac{1}{X^{2}} \frac{d X}{d t} & =\left(r\left(1-\frac{1}{K X}\right)-q \frac{Y}{X}\right) \frac{1}{X} \\
\frac{1}{X} \frac{d Y}{d t} & =\frac{d y}{d t}+\frac{Y}{X^{2}} \frac{d X}{d t}
\end{array} .\right.\right.
$$

$$
Z_{\eta}(X, Y):\left\{\begin{aligned}
\frac{d X}{d t} & =-X\left(r\left(1-\frac{1}{K X}\right)-q \frac{Y}{X}\right) \\
\frac{d Y}{d t} & =X\left(\left(p \frac{1}{X}-c\right) \frac{Y}{X}+\frac{Y}{X^{2}}\left(-X\left(r\left(1-\frac{1}{K X}\right)-q \frac{Y}{X}\right)\right)\right) .
\end{aligned}\right.
$$

Arreglando algebraicamente de llega a

$$
Z_{\eta}(X, Y):\left\{\begin{array}{rl}
\frac{d X}{d t} & =-\left(r\left(X-\frac{1}{K}\right)-q Y\right), \\
\frac{d Y}{d t} & =\frac{1}{X} Y \frac{r+K p-K X c-K X r+K Y q}{K}
\end{array} .\right.
$$

reescalando el tiempo por $t=\operatorname{rac} 1 X \tau$, se obtiene $\frac{d X}{d \tau}=\frac{d X}{d t} \frac{d t}{d \tau}, \frac{d Y}{d \tau}=\frac{d Y}{d t} \frac{d t}{d \tau}$, es decir,

$$
\bar{Z}_{\eta}(X, Y):\left\{\begin{array}{l}
\frac{d X}{d \tau}=K\left(-\left(r\left(X-\frac{1}{K}\right)-q Y\right)\right) X, \\
\frac{d Y}{d \tau}=(r+K p-K X c-K X r+K Y q) Y .
\end{array}\right.
$$

Luego, la matriz Jacobiana del campo vectorial $\bar{Z}_{\eta}(X, Y)$ es

$$
D \bar{Z}_{\eta}(X, Y)=\left(\begin{array}{cc}
r-2 K X r+K Y q & K X q \\
-K Y(c+r) & r+K p-K X c-K X r+2 K Y q
\end{array}\right)
$$

Evaluando la matriz Jacobiana en el punto $(0,0)$, se tiene

$$
D \bar{Z}_{\eta}(0,0)=\left(\begin{array}{cc}
r & 0 \\
0 & r+K p
\end{array}\right) .
$$

Por tanto, $\operatorname{det} D \bar{Z}_{\eta}(0,0)=r(r+K p)>0 \mathrm{y} \operatorname{tr} D \bar{Z}_{\eta}(0,0)=2 r+K p>0$.

De acuerdo al Teorema de la traza y el determinante, se tiene que el punto $(0,0)$ del campo vectorial $\bar{Z}_{\eta}(X, Y)$ es repulsor hiperbólico.

Entonces, el punto $(0,0)$ del campo vectorial $Z_{\eta}(X, Y)$ es un repulsor no-hiperbólico.

Por lo tanto, el punto $(\infty, 0)$ del campo vectorial $Y_{\eta}(x, y)$ es un repulsor no-hiperbólico. 
Observación 1.2. Este resultado implica que las soluciones del sistema nacen en el punto $(\infty, 0)$ o en sus vecindades y el flujo del sistema en el plano de fase tiende hacia la izquierda, en la dirección del eje coordenado $y$.

Matemáticamente, los resultados demostrados para el modelo de Volterra descrito por el sistema (1.1) implican que el modelo de Lotka-Volterra $(L V)$, en que todas las trayectorias son ciclos alrededor de un punto de equilibrio, no es estructuralmente estable. La inclusión de interferencia entre las presas, cambia sustancialmente el comportamiento de las soluciones [18].

2. Modelos derivados. Como es bien sabido, se obtienen nuevos sistemas de EDO, agregando más términos a los modelos básicos o haciendo cambios en la la respuestas funcionales [1] o en la ecuación de crecimiento de los depredadores [21].

En esta sección, presentaremos modelos derivados del modelo de Volterra. Los nuevos modelos pueden tener cambios significativos en sus comportamientos dinámicos, con respecto a los originales, tal como sucede con el modelo de Volterra aqui estudiado, comparado con el modelo de Lotka-Volterra.

2.1. Modelo para la competencia entre los depredadores. La competencia (intraespecífica) entre los depredadores (CED) es incluida en la segunda ecuación del sistema (1.1), obteniendo siguiente sistema

$$
C_{\nu}(x, y):\left\{\begin{aligned}
\frac{d x}{d t} & =\left(r\left(1-\frac{x}{K}\right)-q y\right) x \\
\frac{d y}{d t} & =(-c+p x-f y) y
\end{aligned}\right.
$$

con $\nu=(r, a, q, c, p, f) \in \mathbb{R}_{+}^{6}$ y donde $x=x(t)$ e $y=y(t)$ indican los tamaños de población de presas y depredadores, respectivamente, para $t \geq 0$, como en los modelos anteriores.

En este caso, el término $g(y)=-f y^{2}$, con $f>0$, indica la competencia intraespecifica entre los depredadores.

Observación 2.1. Cuando $f=0$, el sistema (2.1) describe el modelo de Volterra [30], estudiado más arriba.

Los puntos de equilibrio se obtienen considerando $\frac{d x}{d t}=0=\frac{d y}{d t}$; ellos son $(0,0),(K, 0), \mathrm{y}\left(x_{e}, y_{e}\right)$ que satisface las ecuaciones de las isoclinas

$\begin{aligned} y & =\frac{r}{q}\left(1-\frac{x}{K}\right) \text { e } y=\frac{1}{f}(p x-c), \\ \text { es decir, } x_{e} & =\frac{K(c q+f r)}{f r+K p q} \text { e } y_{e}=\frac{K p r-c r}{f r+K p q} .\end{aligned}$

Siguiendo a Colin W. Clark [8], en modelos bioeconómicos, la función $B(y)=-f y$ expresa la interferencia entre los buques pesqueros (hombres como depredadores) que capturan un banco de peces, lo que resulta en una disminución en las tasas de captura.

2.1.1. Propiedades principales del sistema (2.1). Evaluando la matriz Jacobiana se demuestra que $(0,0)$ y $(K, 0)$ son sillas hiperbólicas, si y sólo si, $K p r-c r>0$.

El equilibrio $(K, 0)$ es un atractor, si y sólo si, $K p r-c r<0$; coincide con $\left(x_{e}, y_{e}\right)$, si y sólo si, $K p r-c r=0$, siendo un silla-nodo atractor.

Por tanto, tiene un comportamiento dinámico similar al modelo de Volterra.

El punto de equilibrio $\left(x_{e}, y_{e}\right)$, cuando existe en el interior del primer cuadrante es local asintóticamente estable.

Teorema 2.1. En el sistema (2.1) no existen órbitas periódicas o ciclos.

Demostración: Usando Test o criterio de Bendixson Dulac [8, 10], se debe determinar el signo de la expresión

$$
H(x, y)=\frac{\partial}{\partial x}(P(x, y) g(x, y))+\frac{\partial}{\partial y}(Q(x, y) g(x, y)) .
$$

Considerando $g(x, y)=\frac{1}{x y}$, se obtiene

$$
\begin{aligned}
H(x, y) & =\frac{\partial}{\partial x}\left(\left(r\left(1-\frac{x}{K}\right)-q y\right) \frac{1}{y}\right)+\frac{\partial}{\partial y}\left((p x-c-f y) \frac{1}{x}\right) \\
& =-\frac{1}{K} \frac{r}{y}-\frac{f}{x}<0
\end{aligned}
$$

$H(x, y)$ no puede cambiar de signo,

Luego, se descarta la existencia de ciclos y ciclos límites.

Aplicando el Teorema de Poincaré-Bendixson se obtiene que el punto $\left(x_{e}, y_{e}\right)$ es global asintóticamente estable, si sólo si, $K p r-c r>0$. 
2.2. Competencia entre depredadores generalistas. Es sabido que algunas especies de depredadores consumen un alimento alternativo cuando su presa favorita no está disponible en el medio ambiente común. En esta situación, en el modelo se cambia la tasa de mortalidad en ausencia de presas por una tasa de crecimiento intrínseco positiva.

Luego, el modelo para la competencia entre depredadores (CED) generalistas es descrito por el sistema siguiente

$$
E_{\rho}(x, y):\left\{\begin{aligned}
\frac{d x}{d t} & =\left(r\left(1-\frac{x}{K}\right)-q y\right) x \\
\frac{d y}{d t} & =(s+p x-f y) y
\end{aligned}\right.
$$

con $\rho=(r, a, q, s, p, f) \in \mathbb{R}_{+}^{6}$ y donde $x=x(t)$ e $y=y(t)$ indican los tamaños de población de presas y depredadores, respectivamente, para $t \geq 0$, como en los modelos anteriores.

El sistema (2.2) tiene cuatro puntos de equilibrio son $(0,0),(K, 0),\left(0, \frac{s}{f}\right)$ y $\left(x_{e}, y_{e}\right)$ que satisface las ecuaciones de las isoclinas

$$
y=\frac{r}{q}\left(1-\frac{x}{K}\right) \text { e } y=\frac{s+p x}{f} .
$$

Observamos que:

i) La primera isoclina es una recta de pendiente negativa que pasa por los puntos $\left(0, \frac{r}{q}\right)$ y $(K, 0)$.

ii) La segunda isoclina es una recta de pendiente positiva que pasa por el punto de equilibrio $\left(0, \frac{s}{f}\right)$.

Por lo tanto, hay intersección entres estás rectas, si y sólo si, $\frac{s}{f}<\frac{r}{q}$.

Esta relación paramétrica se puede comprobar en la ecuación que satisface la absisa del punto de equilibrio positivo,

$$
\frac{r}{q}\left(1-\frac{x}{K}\right)-\frac{s+p x}{f}=0,
$$

cuya solución es $x_{e}=\frac{K(f r-q s)}{f r+K p q}$. Luego, $y_{e}=\frac{r}{q}\left(1-\frac{1}{K} \frac{K(f r-q s)}{f r+K p q}\right)=r \frac{s+K p}{f r+K p q}$.

En este caso el nuevo punto de equilibrio sobre el eje vertical tiene fuerte influencia en el comportamiento general del sistema.

2.2.1. Propiedades principales del sistema (2.2). La matrix Jacobiana asociada al sistema es

$$
D E_{\rho}(x, y)=\left(\begin{array}{cc}
-\frac{1}{K}(2 r x-K r+K q y) & -q x \\
p y & s-2 f y+p x
\end{array}\right) .
$$

Con la evaluación en los puntos de equilibrio se tiene que

\section{Lema 2.1.}

a) El punto $(0,0)$ es un repulsor hiperbólico para todo valor de parámetro.

b) El punto $(K, 0)$ es una silla hiperbólica para todo valor de parámetro.

c) El punto de equilibrio $\left(0, \frac{s}{f}\right)$ es

c.1) una silla hiperbólica, si y sólo si, $f r-q s>0$, cuando $\left(x_{e}, y_{e}\right)$ existe en el interior del primer cuadrante.

c.2) un atractor hiperbólico, si y sólo si, $f r-q s<0$, y cuando $\left(x_{e}, y_{e}\right)$ no existe en el interior del primer cuadrante.

c.3) un atractor no-hiperbólico, si y sólo si, $f r-q s=0$, cuando $\left(x_{e}, y_{e}\right)$ coincide con $\left(0, \frac{s}{f}\right)$.

d) El punto $\left(x_{e}, y_{e}\right)$ es atractor local, si y sólo si $\mathrm{fr}-q s>0$.

Demostración: a) En el punto $(0,0)$ se tiene

$$
D E_{\rho}(0,0)=\left(\begin{array}{cc}
r & 0 \\
0 & s
\end{array}\right) \text {. }
$$

Claramente, $\operatorname{det} D E_{\rho}(0,0)>0 \mathrm{y} \operatorname{tr} D E_{\rho}(0,0)>0$.

b) En el punto $(K, 0)$ se tiene

$$
D E_{\rho}(K, 0)=\left(\begin{array}{cc}
-2 r & -q K \\
0 & s+p K
\end{array}\right) \text {. }
$$

Luego, $\operatorname{det} D E_{\rho}(0,0)<0$.

a) En el punto $\left(0, \frac{s}{f}\right)$ se tiene

Es decir,

$$
D E_{\rho}\left(0, \frac{s}{f}\right)=\left(\begin{array}{cc}
\frac{f r-q s}{f} & 0 \\
\frac{p s}{f} & -s
\end{array}\right) \text {. }
$$




$$
\begin{aligned}
& \operatorname{det} D E_{\rho}\left(0, \frac{s}{f}\right)=-s \frac{f r-q s}{f}, \text { (negativo o positivo), y } \\
& \operatorname{tr} D E_{\rho}\left(0, \frac{s}{f}\right)=\frac{f r-q s-f s}{f}<0, \text { cuando } f r-q s<0 .
\end{aligned}
$$

d) En el punto $\left(x_{e}, y_{e}\right)$ se tiene

luego,

$$
D E_{\rho}\left(x_{e}, y_{e}\right)=\left(\begin{array}{cc}
-r \frac{x_{e}}{K} & -q x_{e} \\
p\left(r \frac{K-x_{e}}{q K}\right) & \left(p+\frac{2}{K} \frac{f}{q} r\right) x_{e}+\left(s-2 \frac{f}{q} r\right)
\end{array}\right)
$$

$$
\operatorname{det} D E_{\rho}\left(x_{e}, y_{e}\right)=r x_{e} \frac{(-2 f r-2 K p q) x_{e}+\left(K^{2} p q+2 K f r-K q s\right)}{K^{2} q},
$$

reemplazando, se obtiene que

$$
\operatorname{det} D E_{\rho}\left(x_{e}, y_{e}\right)=r\left(\frac{K(f r-q s)}{f r+K p q}\right) \frac{s+K p}{K},
$$

que depende del signo del factor $f r-q s>0$.

La traza es

$$
\begin{aligned}
\operatorname{trDE} E_{\rho}\left(x_{e}, y_{e}\right) & =\left(p-\frac{1}{K} r+\frac{2}{K} \frac{f}{q} r\right) x+\left(s-2 \frac{f}{q} r\right), \\
& =-r \frac{f r-q s+f s+K f p}{f r+K p q}
\end{aligned}
$$

que es negativa, si y sólo si, $f r-q s>0$.

2.3. Cooperación entre los depredadores. En este caso el sistema

$$
D_{\nu}(x, y):\left\{\begin{aligned}
\frac{d x}{d t} & =\left(r\left(1-\frac{x}{K}\right)-q y\right) x \\
\frac{d y}{d t} & =(-c+p x+f y) y
\end{aligned}\right.
$$

representa un modelo depredador-presa en el cual los depredadores son colaboradores o cooperadores y al juntarse ayudan al crecimiento de la población, con $\nu=(r, K, q, c, p, f) \in \mathbb{R}_{+}^{6}$ y donde $x=x(t) \mathrm{e}$ $y=y(t)$ indican los tamaños de población de presas y depredadores, respectivamente, para $t \geq 0$.

Los puntos de equilibrio del sistema (2.3) son $(0,0),(K, 0),\left(0, \frac{c}{f}\right)$ y $\left(x_{e}, y_{e}\right)$ satisfaciendo la ecuación de las isoclinas:

Luego,

$$
y=\frac{r}{q}\left(1-\frac{x}{K}\right) \text { e } y=\frac{c-p x}{f} \operatorname{con} 1-\frac{x}{K}>0 \text { y } c-p x>0 .
$$

$$
\begin{aligned}
& x_{e}=\frac{K(f r-c q)}{f r-K p q}, \text { con } f r-c q>0 \quad \text { y } \quad f r-K p q>0, \quad \mathrm{y} \\
& y_{e}=r \frac{K p-c}{f r-K p q}, \operatorname{con} K p-c>0 .
\end{aligned}
$$

En el artículo [16], se hace un estudio detallado de este sistema.

2.3.1. Propiedades principales del sistema (2.3). Observamos que se puede aplicar el Criterio de Bendixson-Dulac $[8,10]$, considerando la función $g(x, y)=1$; se obtiene que

$$
\begin{aligned}
H(x, y) & =\frac{\partial}{\partial x}(P(x, y) g(x, y))+\frac{\partial}{\partial y}(Q(x, y) g(x, y)) \\
& =\frac{\partial}{\partial x}\left(\left(r\left(1-\frac{x}{K}\right)-q y\right) 1\right)+\frac{\partial}{\partial y}((p x-c+f y) 1) \\
& =f-\frac{1}{K} r .
\end{aligned}
$$

Por lo tanto, la función $H(x, y)$ puede cambiar de signo, lo cual no descarta la existencia de ciclos.

De hecho, existe una subregion triangular donde existe un punto de equilibrio positivo, que es un centro [16].

Además, el sistema puede tener un equilibrio positivo localmente estable coexistiendo con soluciones que tienden rápidamente al punto $(0, \infty)$ cuando $t \rightarrow \infty$ [16].

Este ejemplo es importante porque existe la creencia generalizada de que en un modelo de interacciones entre dos especies, descritos por sistemas cuadráticos del tipo Kolmogorov, la estabilidad local implica estabilidad global [14].

Existen otras formulaciones matemáticas para modelar la cooperación entre los depredadores para realizar la captura de sus presa (hunting cooperation), como se propone en [29]. 
3. Modelos para el mutualismo y la competencia. Además de la interacción de depredación, existen otras interacciones en la cadenas tróficas o redes de alimentación, como son la competencia entre especies, la simbiosis o mutualismo, el amensalismo, el comensalismo, el pastoreo, y la relación huesped parásito.

3.1. Competencia entre especies. En la naturaleza, se observa frecuentemente que la lucha por la existencia entre dos especies similares, compitiendo por el mismo alimento y por espacios vitales compartidos, casi siempre termina en la completa extinción de una de las especies.

Este fenómeno se conoce como el "principio de exclusión competitiva"[4]. Fue enunciado por primera vez, en una forma ligeramente diferente, por el biólogo inglés Charles Darwin en 1859.

Más precisamente, la competencia ocurre cuando dos o más individuos o especies experimentan una disminución de la aptitud ( $r$ o $K$ reducidos) atribuible a su presencia mutua en un área deteminada [25].

El sistema

$$
C_{\mu}(x, y):\left\{\begin{aligned}
\frac{d x}{d t} & =\left(r\left(1-\frac{x}{K_{1}}\right)-q_{1} y\right) x \\
\frac{d y}{d t} & =\left(s\left(1-\frac{y}{K_{2}}\right)-q_{2} x\right) y
\end{aligned}\right.
$$

describe un modelo para la competencia entre dos especies, donde $x=x(t)$ e $y=y(t)$, representan los tamaños poblacionales de cada especie para $t \geq 0$ (medido en biomasa, número de individuos o densidad); los parámetros son todos positivos, esto es, $\mu=\left(r, K_{1}, q_{1}, s, K_{2}, q_{2}\right) \in \mathbb{R}_{+}^{6} \mathrm{y}$ tienen los siguientes significados ecológicos:

$r$ y $s$ expresan las tasas de crecimiento intrínseco o potencial biótico de cada una de las especie,

$K_{1}$ y $K_{2}$ indican la capacidad de soporte del medio ambiente para cada especie;

$q_{1}$ y $q_{2}$ son las tasas de competencia de cada especie.

Como todas las interacciones descritas anteriormente, el sistema (3.1) está definido en el primer cuadrante $\Omega=\left\{(x, y) \in \mathbb{R}^{2}: x \geq 0, y \geq 0\right\}=\left(\mathbb{R}_{0}^{+}\right)^{2}$.

Los puntos de equilibrio del sistema $(3,1)$ son: $(0,0),\left(K_{1}, 0\right),\left(0, K_{2}\right)$ y $\left(x_{e}, y_{e}\right)$ en la intersección de las isoclinas $y=\frac{r}{q_{1}}\left(1-\frac{x}{K_{1}}\right)$ e $y=K_{2}\left(1-\frac{q_{2}}{s} x\right)$, aunque podría no existir un equilibrio positivo.

El punto $\left(x_{e}, y_{e}\right)$ está en el interior del primer cuadrante, si y sólo si, $x_{e}<\frac{s}{q_{2}}$ y $x_{e}<K_{1}$, pero además la pendiente de las rectas deben ser distintas.

$\mathrm{Si} \frac{r}{q_{1} K_{1}}=\frac{K_{2} q_{2}}{s}$, entonces las rectas son paralelas y no hay intersección entre las isoclinas.

La matriz Jacobiana es

$$
D X_{\mu}(x, y)=\left(\begin{array}{cc}
-\frac{1}{K_{1}}\left(2 r x-r K_{1}+y K_{1} q_{1}\right) & -q_{1} x \\
-q_{2} y & -\frac{1}{K_{2}}\left(2 s y-s K_{2}+x K_{2} q_{2}\right)
\end{array}\right) .
$$

Un análisis más detallado sobre este modelo, se encuentra en el libro de Martin Braun de 1990 [4] y los tipos de competencia se pueden ver en el libro de Dick Neal [25].

3.1.1. Propiedades principales del sistema (3.1). La competencia entre dos especies se caracteriza porque una de las poblaciones interactuantes puede desaparecer, lo que queda explicitado en el Principio de exclusión competitiva, que se enuncia más abajo. En la misma línea de los resultados presentados en las secciones anteriores, se tiene lo siguiente

Lema 3.1. El conjunto $\Lambda=\left\{(x, y) \in \Omega / 0 \leq x \leq K_{1}, 0 \leq y \leq K_{2}\right\}$ es una región positivamente invariante.

Demostración: Claramente los ejes coordenados son conjuntos inavariantes [6], pues el sistema es del tipo Kolmogorov [12].

Considerando $x=K_{1}$, se tiene que $\frac{d x}{d t}=-q_{1} x y<0$ y cualquiera sea el signo de $\frac{d y}{d t}=s\left(1-\frac{y}{K_{2}}\right) y-$ $q_{2} K_{1} y$, las trayectorias entran a la región $\Lambda$.

Analógamente, si $y=K_{2}$, se tiene $\frac{d x}{d t}=-q_{2} x y<0$ y cualquiera sea el signo de $\frac{d x}{d t}=r\left(1-\frac{x}{K_{1}}\right) x-$ $q_{1} x y$, las trayectorias entran a la región $\Lambda$.

Teorema 3.1. (Principio de exclusión competitiva). Supongamos que $K_{1}$ es mayor que $K_{2}$. Entonces, toda solución $x(t)$ e y $(t)$ del sistema (3.1) se aproxima a la solución de equilibrio $\left(K_{1}, 0\right)$ cuando $t$ tiende a infinito. En otras palabras, si las especies son casi idénticas, y el medio ambiente puede albergar más miembros de la primera especie que de la segunda especie, entonces esta última finalmente se extinguirá.

Demostración: Ver libro de M. Braun [4].

Observación 3.1. Si $K_{2}$ es mayor que $K_{2}$. Entonces, toda solución $x(t)$ e y $(t)$ del sistema (3.1) se aproxima a la solución de equilibrio $\left(0, K_{2}\right)$ cuando t tiende a infinito. 
3.2. Modelo para el mutualismo entre dos especies. En la interacción mutualista de dos especies la presencia de cada una de ellas es beneficiosa para la otra. Esta clase de modelos, han sido estudiada en gran medida, y resulta además, ser matemáticamente la más manejable [14].

Según Peter Turchin [30], el mutualismo podría ser la interacción más importante en algunos sistemas de población específicos, pero también afirma que "todos los organismos son consumidores de algo, y la mayoría también son un recurso para otras especies"[30].

El sistema

$$
M_{\nu}(x, y):\left\{\begin{aligned}
\frac{d x}{d t} & =\left(r\left(1-\frac{x}{K_{1}}\right)+q_{1} y\right) x \\
\frac{d y}{d t} & =\left(s\left(1-\frac{y}{K_{2}}\right)+q_{2} x\right) y
\end{aligned}\right.
$$

describe un modelo para el mutualismo, donde $x=x(t)$ e $y=y(t)$, representan los tamaños poblacionales de cada especie para $t \geq 0$; los parámetros son todos positivos, esto es, $\nu=\left(r, K_{1}, q_{1}, s, K_{2}, q_{2}\right) \in$ $\mathbb{R}_{+}^{6}$ y tienen los siguientes significados ecológicos:

$r$ y $s$ expresan las tasas de crecimiento intrínseco o potencial biótico de cada una de las especie,

$K_{1}$ y $K_{2}$ indican la capacidad de soporte del medio ambiente para cada especie;

$q_{1}$ y $q_{2}$ son las tasas de beneficio para cada especie.

Los puntos de equilibrio del sistema (3.2) son $(0,0),\left(K_{1}, 0\right),\left(0, K_{2}\right)$ y $\left(x_{e}, y_{e}\right)$ que satisface la ecuación de las isoclinas

$$
y=\frac{r}{q_{1}}\left(\frac{x}{K_{1}}-1\right) \text { e } y=\frac{K_{2}}{s}\left(s+q_{2} x\right) .
$$

Igualando ambas ecuaciones se obtiene que:

$$
x_{e}=\frac{s K_{1}\left(r+K_{2} q_{1}\right)}{r s-K_{1} K_{2} q_{1} q_{2}} ; \text { por tanto, } y=r K_{2} \frac{s+K_{1} q_{2}}{r s-K_{1} K_{2} q_{1} q_{2}}, \text { con } r s-K_{1} K_{2} q_{1} q_{2}>0 .
$$

La matriz Jacobiana es

$$
D M_{\nu}(x, y)=\left(\begin{array}{cc}
\frac{1}{K_{1}}\left(r K_{1}-2 r x+K_{1} q_{1} y\right) & q_{1} x \\
q_{2} y & \frac{1}{K_{2}}\left(s K_{2}-2 s y+K_{2} q_{2} x\right)
\end{array}\right) .
$$

3.2.1. Propiedades principales del sistema (3.2). Intuitivamente se sabe, que los tamaños poblacionales crecen sin cota porque las especies se benefician recíprocamente.

Lema 3.2. Todo el primer cuadrante $\mathbb{R}_{0}^{+} \times \mathbb{R}_{0}^{+}$es una región positivamente invariante.

Demostración: Cuando $x=K_{1}$, se tiene $\frac{d x}{d t}=q_{1} y K_{1}>0$, y

cuando $y=K_{2}$, se tiene $\frac{d x}{d t}=q_{1} x K_{2}>0$.

Por tanto, las soluciones se alejan de los ejes.

Lema 3.3. Naturaleza de los puntos de equilibrio sobre los ejes

I) El punto $(0,0)$ es un repulsor hiperbólico.

II) El punto $\left(K_{1}, 0\right)$ es una silla hiperbólica.

III) El punto $\left(0, K_{2}\right)$ es una silla hiperbólica.

Demostración: Es inmediata, pues evaluando en los puntos de equilibrio se tiene:

$$
\begin{aligned}
& \text { I) } D M_{\nu}(0,0)=\left(\begin{array}{ll}
r & 0 \\
0 & s
\end{array}\right) . \\
& \text { II) } D M_{\nu}\left(K_{1}, 0\right)=\left(\begin{array}{cc}
-r & K_{1} q_{1} \\
0 & s+K_{1} q_{2}
\end{array}\right) \text {, y } \\
& \text { III) } D M_{\nu}\left(0, K_{2}\right)=\left(\begin{array}{cc}
r+K_{2} q_{1} & 0 \\
K_{2} q_{2} & -s
\end{array}\right) .
\end{aligned}
$$

Teorema 3.2. El punto $\left(x_{e}, y_{e}\right)$ es global asintóticamente estable.

Demostración: Evaluando la matriz Jacobiana en el punto $\left(x_{e}, y_{e}\right)$, reemplazando las correspondientes coordenadas se obtiene:

Luego,

$$
D M_{\nu}\left(x_{e}, y_{e}\right)=\left(\begin{array}{cc}
-r \frac{x_{e}}{K_{1}} & q_{1} x_{e} \\
q_{2} \frac{r}{q_{1}}\left(\frac{x_{e}}{K_{1}}-1\right) & \frac{2 r s K_{1}-2 r s x_{e}+s K_{1} K_{2} q_{1}+K_{1} K_{2} q_{1} q_{2} x_{e}}{K_{2} q_{1} K_{1}}
\end{array}\right) .
$$

$$
\begin{aligned}
\operatorname{det} D M_{\nu}\left(x_{e}, y_{e}\right) & =r x_{e} \frac{\left(2 r s-2 K_{1} K_{2} q_{1} q_{2}\right) x_{e}+\left(K_{1}^{2} K_{2} q_{1} q_{2}-2 r s K_{1}-s K_{1} K_{2} q_{1}\right)}{K_{1}^{2} K_{2} q_{1}} \\
& =K_{1} K_{2} q_{1}\left(s+K_{1} q_{2}\right)
\end{aligned}
$$


Además,

$$
\operatorname{tr} D M_{\nu}\left(x_{e}, y_{e}\right)=-r s \frac{r+s+K_{1} q_{2}+K_{2} q_{1}}{r s-K_{1} K_{2} q_{1} q_{2}}<0 .
$$

Por tanto, el punto $\left(x_{e}, y_{e}\right)$ es local asintóticamente estable.

Como el punto $(0,0)$ es repulsor y los puntos $\left(K_{1}, 0\right)$ y $\left(0, K_{2}\right)$ son sillas, por Teorema de PoincaréBendixson, el único punto que puede ser $\omega$-límite de las trayectorias es $\left(x_{e}, y_{e}\right)$.

La no existencia de ciclos (y ciclos límites) se prueba usando el Test de Bendixson-Dulac con la función $g(x, y)=1$.

Luego, el punto $\left(x_{e}, y_{e}\right)$ es global asintóticamente estable.

4. Modelos para el amensalismo y el comensalismo. El amensalismo y el comensalismo son dos importantes interacciones que están presentes en la naturaleza.

4.1. Modelo para el amensalismo. En la naturaleza existen variados ejemplos de esta interacción, como por ejemplo:

(i) algas planctónicas que liberan una sustancia tóxica, que se concentra en los denominados 'puntos rojos' del océano, provocando la muerte de algunas especies de animales marinos.

(ii) árboles de gran tamaño que impiden la llegada de luz solar a las hierbas que crecen a ras del suelo.

(iii) la antibiosis que es una interacción biológica en que ciertos organismos segregan una sustancia, llamada antibiótico, que provoca la muerte de otros que viven en las inmediaciones. El sistema

$$
A_{\sigma}(x, y):\left\{\begin{aligned}
\frac{d x}{d t} & =\left(r\left(1-\frac{x}{K}\right)-q y\right) x \\
\frac{d y}{d t} & =(s-f y) y
\end{aligned}\right.
$$

describe un modelo para el amensalismo, donde $x=x(t)$ e $y=y(t)$, representan los tamaños poblacionales de cada especie para $t \geq 0$; los parámetros son todos positivos, esto es, $\sigma=(r, K, q, s, f) \in$ $\mathbb{R}_{+}^{5}$ y tienen los mismos significados ecológicos que en el caso de depredación considerando competencia entre los depredadores.

Notamos que la ecuación para el crecimiento de los depredadores es independiente de la población de presas; por lo tanto el sistema podria ser resuelto explícitamente.

El sistema (4.1) tiene sólo dos puntos de equilibrio en el primer cuadrante los cuales son $(K, 0)$ y $\left(K \frac{f r-q s}{r f}, \frac{s}{f}\right)$, con $f r-q s>0$, o sea, $\frac{s}{f}<\frac{r}{q}$.

La matriz Jacobiana es:

$$
D A_{\sigma}(x, y)=\left(\begin{array}{cc}
-\frac{1}{K}(2 r x-K r+K q y) & -q x \\
0 & s-2 f y
\end{array}\right) .
$$

4.1.1. Propiedades principales del sistema (4.1). Con la evaluación de esta matriz en los puntos de equilibrio se tiene que

I) El punto $(K, 0)$ es silla hiperbólica, pues

$$
D A_{\sigma}(K, 0)=\left(\begin{array}{cc}
-r & -K q \\
0 & s
\end{array}\right)
$$

II) El punto $\left(K \frac{f r-q s}{r f}, \frac{s}{f}\right)$ es atractor hiperbólico, puesto que:

$$
\begin{aligned}
& D A_{\sigma}\left(K \frac{f r-q s}{r f}, \frac{s}{f}\right)=\left(\begin{array}{cc}
-\frac{f r-q s}{f} & -q x \\
0 & -s
\end{array}\right), \text { con } \\
& \operatorname{det} D A_{\sigma}\left(K \frac{f r-q s}{r f}, \frac{s}{f}\right)=\frac{f r-q s}{f} s>0, \mathrm{y} \\
& \operatorname{tr} D A_{\sigma}\left(K \frac{f r-q s}{r f}, \frac{s}{f}\right)=-\frac{f r-q s}{f}-s<0 .
\end{aligned}
$$

4.2. Modelo para el comensalismo. Algunos ejemplos de esta interacción que se ven en la naturaleza son:

(i) semillas que se dispersan adheridas al pelaje de animales;

(ii) organismos (ácaros, bacterias, etc.) que viven en la piel de los animales pero no dañan al huésped;

(iii) un nido de pájaro en un árbol; o

(iv) aves que se alimenta de manera más eficiente subida al lomo de ciertos animales.

El siguiente sistema

$$
C_{\kappa}(x, y):\left\{\begin{aligned}
\frac{d x}{d t} & =\left(r\left(1-\frac{x}{K}\right)+q y\right) x \\
\frac{d y}{d t} & =(s-f y) y
\end{aligned}\right.
$$


describe un modelo para el amensalismo, donde $x=x(t)$ e $y=y(t)$, representan los tamaños poblacionales de cada especie para $t \geq 0$; los parámetros son todos positivos, esto es, $\kappa=(r, K, q, s, f) \in \mathbb{R}_{+}^{5}$ y tienen los mismos significados ecológico que en el caso de mutualismo, pero considerando competencia interespecífica entre presas y depredadores.

También la ecuación para el crecimiento de los depredadores es independiente de la población de presas; luego, el sistema puede ser resuelto explícitamente.

El sistema (4.2) tiene sólo dos puntos de equilibrio en el primer cuadrante los cuales son $(K, 0)$ y $\left(K \frac{f r+q s}{r f}, \frac{s}{f}\right)$.

La matriz Jacobiana es:

$$
D C_{\kappa}(x, y)=\left(\begin{array}{cc}
\frac{1}{K}(K r-2 r x+K q y) & -q x \\
0 & s-2 f y
\end{array}\right) .
$$

4.2.1. Propiedades principales del sistema (4.2). Evaluando la matriz Jacobiana en los puntos de equilibrio se tiene que

I) El punto $(K, 0)$ es silla hiperbólica, pues

$$
D C_{\kappa}(K, 0)=\left(\begin{array}{cc}
-r & -K q \\
0 & s
\end{array}\right) .
$$

II) El punto $\left(K \frac{f r+q s}{r f}, \frac{s}{f}\right)$ es atractor hiperbólico, puesto que:

$$
\begin{aligned}
& D C_{\kappa}\left(K \frac{f r-q s}{r f}, \frac{s}{f}\right)=\left(\begin{array}{cc}
-\frac{f r+q s}{f} & -q x \\
0 & -s
\end{array}\right), \text { con } \\
& \operatorname{det} D C_{\kappa}\left(K \frac{f r-q s}{r f}, \frac{s}{f}\right)=\frac{f r+q s}{f} s>0, \mathrm{y} \\
& \operatorname{tr} D C_{\kappa}\left(K \frac{f r-q s}{r f}, \frac{s}{f}\right)=-\frac{f r+q s}{f}-s<0 .
\end{aligned}
$$

5. Sistemas cuadráticos del tipo Kolmogorov. Los sistemas polinomiales cuadráticos del tipo Kolmogorov $[12,14]$ son de la forma

$$
K_{\sigma}(x, y):\left\{\begin{aligned}
\frac{d x}{d t} & =\left(r_{1}+a_{11} x+a_{12} y\right) x \\
\frac{d y}{d t} & =\left(r_{2}+a_{21} x+a_{22} y\right) y
\end{aligned}\right.
$$

con parámetros reales, esto es, $\sigma=\left(r_{1}, a_{11}, a_{12}, r_{2}, a_{21}, a_{22}\right) \in \mathbb{R}^{6}$ [27]. Claramente, los ejes son conjuntos invariantes, pero no necesariamente un sistema de la forma (5.1) representa un modelo de interacción entre dos especies.

Matemáticamente ha sido estudiado y existen más de 140 comportamientos dinámicos (retratos de fase globales) diferentes en todo el plano de fase $\mathbb{R}^{2}[27]$.

En forma natural surgen las siguientes preguntas

1) ¿Que condiciones deben cumplir los parámetros para que el sistema represente una interacción entre dos especies?

2) ¿Que condiciones deben cumplir los parámetros para que el sistema represente una interacción de competencia?

3) ¿Que condiciones deben cumplir los parámetros para que el sistema tenga al menos un punto de equilibrio en el interior del primer cuadrante?

4) ¿Que condiciones deben cumplir los parámetros para que el sistema tenga al menos un punto de equilibrio localmente estable al interior del primer cuadrante?

5) ¿Que condiciones deben cumplir los parámetros para que el sistema tenga al menos un punto de equilibrio globalmente estable al interior del primer cuadrante?

6) ¿Cuantos puntos de equilibrio del sistema (5.1) o singularidades del campo vectorial $K_{\sigma}(x, y)$ existen en el primer cuadrante?

Algunas de esas cuestiones han sido respondidas en el libro de Goh [14].

Asumiendo que existe mortalidad denso-dependiente en cada especie, esto es $a_{11}$ y $a_{22}<0$, los puntos de equilibrio del sistema (5.1) o singularidades de campo vectorial $K_{\sigma}(x, y)$ son $(0,0),\left(-\frac{r_{1}}{a_{11}}, 0\right)$ y $\left(0,-\frac{r_{2}}{a_{22}}\right)$, además del punto $\left(x_{e}, y_{e}\right)$, que satisface las ecuaciones de las isoclinas,

$$
r_{1}+a_{11} x+a_{12} y=0 \text {, }
$$

es decir,

$$
r_{2}+a_{21} x+a_{22} y=0
$$


Luego,

$$
y=-\frac{r_{1}+a_{11} x}{a_{12}} \text { y } y=-\frac{r_{2}+a_{21} x}{a_{22}} .
$$

$$
x_{e}=\frac{r_{2} a_{12}-r_{1} a_{22}}{a_{11} a_{22}-a_{12} a_{21}} ; \text { por lo tanto, } y_{e}=\frac{r_{1} a_{21}-r_{2} a_{11}}{a_{11} a_{22}-a_{12} a_{21}},
$$

con

$$
\begin{aligned}
& a_{11} a_{22}-a_{12} a_{21}>0 . r_{2} a_{12}-r_{1} a_{22}>0 \text { y } r_{1} a_{21}-r_{2} a_{11}>0, \text { o bien } \\
& a_{11} a_{22}-a_{12} a_{21}<0, r_{2} a_{12}-r_{1} a_{22}<0, \text { y } r_{1} a_{21}-r_{2} a_{11}<0 .
\end{aligned}
$$

Notamos que estas restricciones corresponden al determinante de la matriz de coeficientes de la interacción,

Para que la primera población crezca debe asumirse que $r_{1}>0$.

Siguiendo el libro de B-S Goh [14], se tiene el siguiente

Teorema 5.1. Condiciones suficientes para la estabilidad global en el modelo (5.1) describiendo una interacción de dos especies son:

1. Existe un equilibrio positivo $\left(x_{e}, y_{e}\right)=\left(\frac{r_{2} a_{12}-r_{1} a_{22}}{a_{11} a_{22}-a_{12} a_{21}}, \frac{r_{1} a_{21}-r_{2} a_{11}}{a_{11} a_{22}-a_{12} a_{21}}\right)$, con $a_{11} a_{22}-a_{12} a_{21}>0$, $r_{2} a_{12}-r_{1} a_{22}>0$ y $r_{1} a_{21}-r_{2} a_{11}>0$,

2. el equilibrio positivo es localmente estable, es decir, detD $K_{\sigma}\left(x_{e}, y_{e}\right)>0$ y $\operatorname{trD} K_{\sigma}\left(x_{e}, y_{e}\right)<0$,

3. cada especie sustenta una mortalidad denso-dependiente debido a interacciones intraespecíficas, es decir, $a_{11}$ y $a_{22}<0$.

Demostración: La matriz Jacobiana del sistema es

$$
\begin{aligned}
& D K_{\sigma}\left(x_{e}, y_{e}\right)=\left(\begin{array}{cc}
r_{1}+2 a_{11} x_{e}+a_{12} y_{e} & a_{12} x_{e} \\
a_{21} y_{e} & r_{2}+a_{21} x_{e}+2 a_{22} y_{e}
\end{array}\right), \\
& \operatorname{det} D K_{\sigma}\left(x_{e}, y_{e}\right)=\frac{\left(r_{1} a_{21}-r_{2} a_{11}\right)\left(r_{2} a_{12}-r_{1} a_{22}\right)}{a_{11} a_{22}-a_{12} a_{21}} . \\
& \operatorname{det} D K_{\sigma}\left(x_{e}, y_{e}\right)>0 \text {, si y solo si, } x_{e}, y_{e}>0 \text { o bien, } x_{e} y_{e}\left(a_{11} a_{22}-a_{12} a_{21}\right)>0 .
\end{aligned}
$$

Además,

$$
\operatorname{tr} D K_{\sigma}\left(x_{e}, y_{e}\right)=\frac{\left(a_{11}\left(r_{2} a_{12}-r_{1} a_{22}\right)+a_{22}\left(r_{1} a_{21}-r_{2} a_{11}\right)\right)}{a_{11} a_{22}-a_{12} a_{21}}=a_{11} x_{e}+a_{22} y_{e} .
$$

Luego, $\operatorname{tr} D K_{\sigma}(x, y)<0$, si y sólo si, $a_{11}$ y $a_{22}<0$.

Eligiendo una función de Lyapunov adecuada se demuestra la estabilidad global (ver el texto de Goh página 68) [14].

Además, evaluando la matriz Jacobiana en cada uno de esos puntos se tiene:

1. $D K_{\sigma}(0,0)=\left(\begin{array}{cc}r_{1} & 0 \\ 0 & r_{2}\end{array}\right)$.

(a) Asumiendo que $r_{1}>0$ y $r_{2}>0$, el punto $(0,0)$ es repulsor.

(b) Asumiendo que $r_{1}>0$ y $r_{2}<0$, el punto $(0,0)$ es silla hiperbólica.

2. $D K_{\sigma}\left(-\frac{r_{1}}{a_{11}}, 0\right)=\left(\begin{array}{cc}r_{1}+2 a_{11}\left(-\frac{r_{1}}{a_{11}}\right) & a_{12}\left(-\frac{r_{1}}{a_{11}}\right) \\ 0 & r_{2}+a_{21}\left(-\frac{r_{1}}{a_{11}}\right)\end{array}\right)$.

(a) Asumiendo que $r_{1}<0$ y $a_{211}<0$, el punto no está en el primer cuadrante y no tiene sentido ecológico.

(b) Asumiendo que $r_{1}>0$ y $a_{11}<0$, el punto $\left(-\frac{r_{1}}{a_{11}}, 0\right)$ está en el primer cuadrante.

$$
\text { Luego, } \operatorname{det} D K_{\sigma}\left(-\frac{r_{1}}{a_{11}}, 0\right)=\frac{r_{1}}{a_{11}}\left(r_{1} a_{21}-r_{2} a_{11}\right) \text {. }
$$

(c) Si $r_{1} a_{21}-r_{2} a_{11}>0$, la naturaleza de depende de la traza

$$
\operatorname{tr} D K_{\sigma}\left(-\frac{r_{1}}{a_{11}}, 0\right)=r_{2}-r_{1}-\frac{r_{1}}{a_{11}} a_{21}=-\frac{r_{1} a_{11}+r_{1} a_{21}-r_{2} a_{11}}{a_{11}}
$$

(d) Si $r_{1} a_{21}-r_{2} a_{11}<0$, entonces $\operatorname{det} D K_{\sigma}\left(-\frac{r_{1}}{a_{11}}, 0\right)<0$, y el punto es silla hiperbólica.

3. $D K_{\sigma}\left(0,-\frac{r_{2}}{a_{22}}\right)=\left(\begin{array}{cc}r_{1}+a_{12}\left(-\frac{r_{2}}{a_{22}}\right) & 0 \\ a_{21}\left(-\frac{r_{2}}{a_{22}}\right) & r_{2}+2 a_{22}\left(-\frac{r_{2}}{a_{22}}\right)\end{array}\right)$,

(a) Asumiendo que $r_{2}<0$ y $a_{22}<0$, o bien, $r_{2}>0$ y $a_{22}>0$, el punto no está en el primer cuadrante y no tiene sentido ecológico.

(b) Asumiendo que $r_{2}>0$ y $a_{22}<0$, el punto está en el primer cuadrante.

Luego, $\operatorname{det} D K_{\sigma}\left(0,-\frac{r_{2}}{a_{22}}\right)=-\frac{r_{2}}{a_{22}}\left(r_{1} a_{22}-r_{2} a_{12}\right)$.

Si $r_{1} a_{22}-r_{2} a_{12}<0$, entonces $\operatorname{det} D K_{\sigma}\left(0,-\frac{r_{2}}{a_{22}}\right)<0$, y el punto es silla hiperbólica. 
Si $r_{1} a_{22}-r_{2} a_{12}>0$, la naturaleza del punto $\left(0,-\frac{r_{2}}{a_{22}}\right)$ depende de la traza

$\operatorname{tr} D K_{\sigma}\left(0,-\frac{r_{2}}{a_{22}}\right)=\frac{r_{1} a_{22}-r_{2} a_{12}-r_{2} a_{22}}{a_{22}}$.

Es decir, depende de $T=\left(r_{1} a_{22}-r_{2} a_{12}\right)-r_{2} a_{22}$.

Notamos que si $r_{2}<0$ y $a_{22}>0$, el punto está en el primer cuadrante, pero no está en las condición del Teorema sobre la interferencia intraespecifica.

En este caso se tiene una situación similar a iv.b, es decir, $\operatorname{det} D K_{\sigma}\left(0,-\frac{r_{2}}{a_{22}}\right)=-\frac{r_{2}}{a_{22}}\left(r_{1} a_{22}-r_{2} a_{12}\right)$.

Si $r_{1} a_{22}-r_{2} a_{12}<0$, entonces $\operatorname{det} D K_{\sigma}\left(0,-\frac{r_{2}}{a_{22}}\right)<0$, y el punto es silla hiperbólica.

Si $r_{1} a_{22}-r_{2} a_{12}>0$, la naturaleza de depende de la traza

$\operatorname{tr} D K_{\sigma}\left(0,-\frac{r_{2}}{a_{22}}\right)=\frac{r_{1} a_{22}-r_{2} a_{12}-r_{2} a_{22}}{a_{22}}$.

Luego, la naturaleza de los puntos de equilibrio sobre los ejes está supeditada a la existencia del punto de equilibrio positivo y a las condiciones establecidas en el enunciado del teorema.

\section{Discusión.}

1. En este documento mostramos las propiedades del modelo de depredación de Volterra [30], usando una función de Lyapunov adecuada para mostrar que el único punto de equilibrio positivo [14], cuando existe, es global asintóticamente estable y no existen ciclos límites.

Esto demuestra que el sistema de Volterra es estructuralmente estable [6], dado que para un gran conjunto de parámetros $(p K-c>0)$ el sistema mantiene sus propiedades, en particular el punto de equilibrio $\left(\frac{c}{p}, y_{e}\right)$, con $y_{e}=\frac{r}{q}\left(1-\frac{c}{p K}\right)$.

En este caso, las poblaciones de presas y depredadores coexisten alrededor del punto $\left(\frac{c}{p}, y_{e}\right)$. Si se perturban los tamaños poblacionales en una pequeña cantidad (por factores exógenos a la interacción) las trayectorias vuelven a este punto.

Cuando $p K-c<0$, el punto de equilibrio $(K, 0)$ es atractor, implicando que la población de depredadores se extingue. Para que esto acontezca, debe cumplirse la relación $\frac{c}{p}>K$, lo cual indica que la tasa de muerte natural $c$, debe ser mucho mas grande que la tasa de reproducción $p$, cuestión que puede suceder con frecuencia en la naturaleza.

Un resultado importante en este modelo es que no existen bifurcaciones de ninguna clase y se puede afirmar que el sistema es estructuralmente estable. El único cambio posible es el señalado anteriormente, en que el punto $(K, 0)$, puede cambiar de estabilidad.

En el modelo de Volterra, como en muchos modelos propuestos para la depredación [17, 20], se asume implícitamente que el depredador es especialista y por lo tanto, cuando la población se reduce, los depredadores tendrán dificultad para que su población crezca.

Una conclusión importante obtenida en las dinámicas de la mayoría de los modelos derivados que aquí se presentan es la existencia de un punto de equilibrio positivo localmente estable que es también globalmente estable. Esto no sucede en el sistema describiendo la colaboración o cooperación entre los depredadores, donde existen soluciones que tienden al punto $(0, \infty)$, coexistiendo con un punto de equilibrio positivo localmente estable [16].

2. El problema de determinar la cantidad de ciclos límites que pueden bifurcar de un centro-foco [6] es una problema abierto en sistemas planares de Ecuaciones Diferenciales Ordinarias [11], aún en el caso que las funciones de lado derecho del sistema sean polinomios [13]. En particular, en modelos de depredación también lo es [9], aunque hay varios trabajos que tratan de demostrar la existencia y unicidad de un único ciclo límite en modelos más generales como el aquí presesentado [17].

A partir de los trabajos de K. S. Cheng [5], muchos autores han tratado de generalizar los resultados para la existencia y unicidad de ciclos límites, en especial para los modelos del tipo Gause [17, 20]. Una manera para determinar la cantidad de ciclos límites rodeando un punto de equlibrio es mediante el cálculo de las cantidades de Lyapunov [6]

3. En los sistemas cuadráticos del tipo Kolmogorov, también se ha estudiado el problema de la existencia de ciclos límites, y se ha demostrado que no existen órbitas cerradas aisladas.

Pero como se muestra en [16], en modelos que describen la cooperación de los depredadores para capturar sus presas favoritas (hunting harvesting) existen condiciones en los parámetros, para las cuales el único punto de equilibrio positivo es un centro (o centro foco), como sucede también en el modelo original de Lotka-Volterra.

Debemos advertir sin embargo, no todos los modelos de interacción entre dos especies son del tipo Kolmogorov. Por ejemplo, si se considera el uso de una cantidad constante de presas en refugio $x_{r}=\gamma$ [23], la cantidad de presas disponible a ser depredadas es $x-\gamma$. Luego, al reemplazar en 
la primera ecuación del modelo de Volterra, no es posible factorizar la variable $x$.

4. En muchos modelos a estudiar, es conveniente primero efectuar una reparametrización y un reescalamiento del tiempo con el objeto de facilitar los cálculos, los que se realizan para un sistema que es topológícamente (cualitativamente) equivalente al sistema original.

Variadas formas para modelar la interacción depredador-presa han sido propuestas, diferente a los modelos de tipo Gause [12, 20], como son los modelos del tipo Leslie-Gower [21]. En todos ellos pueden considerarse diferentes respuestas funcionales [28] o distintas funciones de crecimiento de presas o depredadores [30], expresadas a su vez por distintas formulaciones matemáticas.

La inclusión de diversos fenómenos ecológicos, como el efecto Allee [19], el uso de refugio por una fracción de la población de presas [23], el comportamiento de rebaño, la formación de grupos de defensa [28], la agregación de las especies [8], etc., permite la proposición de modelos descritos por EDO no lineales, para los cuales no existe una clasificación general.

Hacemos presente que los resultados obtenidos son válidos, porque se ha efectuado un análisis coherente y adecuado. Sin embargo, tienen limitaciones pues existen restricciones implícitas en los modelos como son:

- la población es homogénea (todos los individuos son idénticos);

- la población está aislada (no hay inmigración ni emigración);

- el hábitat es invariante (la cantidad de recursos y las condiciones de vida no están influenciadas por factores externos ni por la población misma);

- la población es muy grande (los eventos estocásticos y las fluctuaciones pueden despreciarse), ver [18] página 7.

De cualquier manera, los nuevos modelos que se propongan deben ser utiles, simples y creibles [2].

ORCID and License

Eduardo González-Olivares https://orcid.org/0000-0003-3907-0076

Alejandro Rojas-Palma https://orcid.org/0000-0002-5837-1571

This work is licensed under the Creative Commons - Attribution 4.0 International (CC BY 4.0)

\section{Referencias}

[1] Bazykin AD. Nonlinear Dynamics of interacting populations. Singapore: World Scientific Publishing Co. Pte. Ltd. 1998.

[2] Berryman AA, Gutierrez AP, Arditi R. Credible, parsimonious and useful predator-prey models - A reply to Abrams, Gleeson and Sarnelle, Ecology. 1995 76:1980-1985.

[3] Birkhoff G, Rota GS. Ordinary Differential Equations (4th ed.) New York: John Wiley \& Sons; 1989.

[4] Braun M. Ecuaciones diferenciales y sus aplicaciones. California: Grupo Editorial Iberoamérica; 1990.

[5] Cheng KS. Uniqueness of a limit cycle for a predator-prey system, SIAM J. on Math. Anal. 1981; 12:541-548.

[6] Chicone C. Ordinary differential equations with applications (2nd edition), New YorK: Springer; Texts in Applied Mathematics $34 ; 2006$.

[7] Clark CW. Bioeconomic modelling and fisheries managements. New York: John Wiley and Sons; 1985.

[8] Clark CW. Mathematical Bioeconomic: The optimal management of renewable resources, (second edition). New York: John Wiley and Sons; 1990.

[9] Coleman CS. Hilbert's 16th. Problem: How Many Cycles? In: M. Braun, CS. Coleman and D. Drew (Ed). Differential Equations Model, Springer Verlag. 1983; 279-297.

[10] Edelstein-Keshet L. Mathematical Models in Biology. SIAM; Classics in Applied Mathematics, 46; 2005.

[11] Dumortier F, Llibre J, Artés JC. Qualitative theory of planar differential systems. Berlin: Springer-verlag; 2006.

[12] Freedman HI. Deterministic Mathematical Model in Population Ecology. New York: Marcel Dekker; 1980.

[13] Gaiko VA. Global Bifurcation theory and Hilbert sixteenth problem, Mathematics and its Applications 559. New York: Kluwer Academic Publishers; 2003.

[14] Goh B-S. Management and Analysis of Biological Populations. New York: Elsevier Scientific Publ. Co.; 1980.

[15] González-Olivares E y Mena-Lorca J. Análisis cualitativo de un modelo de pesquerías de acceso abierto. Invest. Mar., Valparaíso. $1994 ; 22: 3-11$.

[16] González-Olivares E, Valenzuela-Figueroa S. and Rojas-Palma A. A simple Gause type predator-prey model considering social predation, Mathematical Methods in the Applied Sciences, 2019; 42:5668-5686.

[17] Hasík K. On a predator-prey system of Gause type. J. of Mathematical Biology. 2010(1):60:59-74.

[18] Iannelli M, Pugliese A. An Introduction to Mathematical Population Dynamics. Italia: Springer; 2014.

[19] Kot M. Elements of Mathematical Ecology. Cambridge: Cambridge University Press; 2003.

[20] Kuang Y, Freedman HI. Uniqueness of limit cycles in Gause-type models of predator-prey systems. Mathematical Biosciences, 1988; 88:67-84.

[21] Leslie PH. Some further notes on the use of matrices in population mathematics, Biometrica. 1948; 35:213-245.

[22] May RM. Stability and complexity in model ecosystems (2nd edition), Princeton: Princeton University Press; 2001.

[23] Maynard Smith J. Models in Ecology, New York: University Press, 1974.

[24] Murray JD. Mathematical Biology I. An Introduction, (3rd ed.), New York: Springer; 2002.

[25] Neal D. Introduction to Population Biology. Cambridge: Cambridge University Press; 2003.

[26] Perko L. Differential equations and dynamical systems (3rd ed.) New York: Springer; 2001.

[27] Schlomiuk D, Vulpe N. Global classification of the planar Lotka-Volterra differential systems according to their configurations of invariant straight lines, J. of Fixed Point Theory and Applications, 2010; 8:177-245. 
[28] Taylor RJ. Predation. London: Chapman and Hall; 1984.

[29] Teixeira Alves M, Hilker FM. Hunting cooperation and Allee effects in predators, J. of Theoretical Biology, 2017; 419: 13-22.

[30] Turchin P. Complex population dynamics. A theoretical/empirical synthesis, Monographs in Population Biology 35. Princeton: Princeton University Press; 2003. 